\title{
A GENERAL PRACTICE PERSPECTIVE OF PANDEMIC INFLUENZA
}

\author{
Michael G Moore \\ Central Sydney Division of General Practice
}

\section{ABSTRACT}

During an influenza pandemic, general practice will inevitably be involved at the front line; however, the nature of the role is likely to vary between jurisdictions and between metropolitan and rural locations. While most of the plans for general practice are still in evolution, measures that general practices can take to prepare for a pandemic include: reviewing the practice's infection control measures; ensuring all at-risk patients are immunised against seasonal influenza and pneumococcus; routinely advising all patients with viral upper respiratory tract infections on infection control techniques; connecting the practice to broadband Internet to aid communication; employing a practice nurse; and appointing a practice pandemic coordinator. General practices should start their preparations now.

What will the role of general practice be in the management of pandemic influenza in Australia? Like so many things in medicine, the answer is 'It depends'. It depends on some unpredictable variables such as the virulence and infectivity of the virus and the effectiveness of preventive and curative interventions. It also depends on variables we can shape and influence, such as the readiness, responsiveness and capacity of Australia's border protection and health systems. If there is good coordination and quick and appropriate responses from the agencies that protect our borders, from the public health system, and from general practice, we may be able to attenuate a future influenza pandemic in Australia, even if a pandemic were to strike other countries in our region.

Many of the guidelines for general practice are still in evolution and many of the plans will vary between the jurisdictions and between metropolitan and rural locations; nonetheless we can still picture what the role of general practice might be and what general practices can do to prepare for the possible pandemic phases. And even if a pandemic never eventuates, most of the preparations for a pandemic are still useful things to do. This article describes the role of general practice through the various phases of a pandemic and provides a framework to assist general practices to prepare.

\section{THE ROLE OF GENERAL PRACTICE IN SURVEILLANCE}

Effective disease surveillance systems help to protect Australia from the spread of evolving viruses. These systems give early warning about disease outbreaks and allow an early and rapid response. Some general practices already play a role in influenza surveillance in NSW by contributing reports of influenza-like illness to either the NSW Sentinel General Practice Scheme or the Australian Sentinel Practice Research Network. The role of general practice in the surveillance of pandemic influenza will be clarified as surveillance guidelines are reviewed.

\section{THE CONTAINMENT PHASE}

Surveillance of travelers entering Australia will not necessarily prevent the entry of evolving influenza viruses into Australia, particularly as people infected with influenza can shed the virus in the 24 hours before they become symptomatic. Should a pandemic influenza strain enter Australia, the initial goal will be to contain it. Recent modelling commissioned by the Commonwealth Department of Health and Ageing suggests that if a containment strategy is vigorously pursued the virus could be contained until a vaccine is available. ${ }^{1}$

NSW Health will inform all NSW general practices and divisions of general practice by fax immediately when the containment phase begins. In this phase, the role of general practice will be to rapidly screen potential presenting cases and refer them without delay to the public health system for diagnosis, treatment and contact tracing. In NSW, general practitioners already work closely with their local public health units. Management of pandemic influenza patients and their contacts will include antiviral medication (either as treatment or post-exposure prophylaxis) and home quarantine. Area health services will arrange antiviral medication for patients and contacts and will work within emergency management systems to provide home support to people in home quarantine. General practice workers who are exposed to a case of pandemic influenza will be provided with post exposure prophylaxis. Updates and additional information will be made available on the NSW Health website.

At the same time, NSW Health hospitals will be activated to screen and assess potentially infected people who present in influenza assessment areas. ${ }^{2}$ These areas will initially be located at NSW Health emergency departments and multipurpose services; however, more locations will be made available should the pandemic progress. Where these assessment areas are in place, potentially infected people will be able to bypass general practice, allowing practices to concentrate on their essential work of providing care for the broader burden of disease. This arrangement will minimize the risk of exposure for general practitioners, general practice staff and their non-influenza patients.

In rural and remote areas where local influenza assessment areas are not planned, existing primary care services will be advised to split their practice location into two areas — influenza and non-influenza — or to pool resources with a neighbouring practice and have separate influenza 
and non-influenza practices. General practices should consult with their local area health service to find out if this applies to them.

\section{Immunisation}

Estimates vary as to the length of time required to develop a vaccine to a newly evolved influenza virus. Australian Government contracts are currently in place with the domestic vaccine manufacturer CSL Limited and with the French manufacturer Sanofi Pasteur. The logistics of a national immunisation program are still to be described and, depending on local arrangements, general practice may or may not have a role in what will be a very rapid population-based immunisation program. In NSW, however, the program will be organised within the public health system.

\section{THE MAINTENANCE OF SOCIAL FUNCTIONING PHASE}

Should a pandemic influenza strain escape containment the goal will be to minimise the transmission rate and the disruption to the community.

In most areas of Australia just under two per cent of the population presents to general practice on any one day. At the peak of a full-blown pandemic, up to one per cent of the population in an area could become ill on any one day. In order to maintain normal general practice services, in NSW general practices will be able to direct most of these additional patients to NSW Health influenza assessment areas. General practices will need good lines of communication with their local area health services and divisions of general practice to keep up to date on locations of these, and on the case descriptions and referral criteria (if any) required for patients to attend them.

\section{PREPARING FOR AN INFLUENZA PANDEMIC}

The list below provides a framework through which general practices may approach planning for a pandemic. A number of useful references that provide greater detail are also listed in this article.

\section{Get organised}

Appoint a practice pandemic coordinator to approach the preparation and planning in your practice in an organised way.

\section{Public health measures}

Regularly and comprehensively vaccinate all your practice's older and at risk patients against both seasonal influenza and pneumococcus. Impress on all patients with viral upper respiratory tract infections the need for cough etiquette and social distancing and the need to wash their hands.

\section{Establish your practice's communication lines}

Get connected to broadband Internet. In a pandemic, case descriptions, treatment protocols and logistics may vary from day to day and you will need to be aware of these changes. Mail services may be unreliable in periods of high absenteeism. Get used to reporting notifiable diseases to your local public health unit as a matter of course. Use the forms built into your prescribing package, download the forms from the NSW Health website (www.health.nsw.gov. $\mathrm{au} /$ public-health/forms) or ring and notify your local public health unit over the phone. Keep up to date-read your local division of general practice newsletter and talk to them about the preparations being made in your community.

\section{Plan for business continuity}

At the peak of a pandemic, absentee rates in the general community could be as high as 30 per cent. Among health care professionals, absentee rates may be even higher. Absenteeism occurs not only through personal illness but also through the need to care for sick relatives and, when schools are closed, to care for children. There is also likely to be voluntary absenteeism to enable voluntary social distancing, and some general practice staff may be rostered to work at influenza assessment areas-so plan now for how your practice would operate in a time of reduced staffing. Talk to neighbouring practices about pooling resources. If you are thinking of employing a practice nurse (or two) for your practice, do it now. Practice nurses enhance the quantity and quality of care for your patients, and should a pandemic eventuate they will be a very useful resource.

\section{Plan your practice infection control measures}

Make sure all your staff know how to fit and remove personal protective equipment, including a gown, mask, protective eyewear and gloves, correctly, and in the correct order. Although most potentially infected patients in NSW will be advised to bypass general practice, some will still present. In a pandemic, hand washing facilities, masks and other personal protective equipment will need to be available for both staff (P2 mask) and patients (surgical mask). Facilities must also be available to disinfect surfaces contacted by potentially infected patients. Comprehensive infection control guidelines are available on the web; see www.health.gov.au/internet/wcms/publishing.nsf/Content/ phd-pandemic-resources.htm and www.health.gov.au/ internet/wcms/publishing.nsf/content/pubhlth-pandemicgp.htm

\section{Work out your supply chains for essential materials}

Your local area health service will coordinate distribution of personal protective equipment and prophylactic antivirals for staff (when required) to general practices in affected areas. Find out from your division of general practice or area health service how this will be managed in your area.

\section{Stay up to date}

The Medical Journal of Australia released a special influenza pandemic preparedness issue in November 2006 (see: (www.mja.com.au/public/issues/185_10_201106/ suppl_contents_201106.html). Early in 2007 the Australian Management Plan for Pandemic Influenza will have what 
promises to be a very comprehensive updated primary care annex, (see www.health.gov.au/internet/wcms/publishing. nsf/Content/phd-pandemic-plan.htm). Make sure you, or your practice pandemic coordinator, review these resources.

\section{CONCLUSION}

Many of the guidelines and plans for an influenza pandemic are still evolving and plans for general practice vary between jurisdictions. Nonetheless, we can picture the role of general practice and what general practices can do to prepare for the possible pandemic phases. Remember, most of the preparations for a pandemic are useful things to do even if a pandemic never eventuates.

\section{REFERENCES}

1. Becker N, Glass K, Barnes B, Caley P, Philp D, McCaw et al. 'Using mathematical models to assess responses to an outbreak of an emerged viral respiratory disease'. Unpublished research paper, Canberra, 2006.

2. NSW Department of Health. 'Hospital response to pandemic influenza. Part 1: Emergency department response'. Unpublished paper (planned release in early 2007), Sydney, 2006. 